\title{
The hot non-perturbative gluon plasma is an almost ideal colored liquid
}

\author{
A. Peshier and W. Cassing \\ Institut für Theoretische Physik, Universität Giessen, 35392 Giessen, Germany
}

(Dated: October 11, 2018)

\begin{abstract}
We study properties of a gluon plasma above the critical temperature $T_{c}$ in a generalized quasiparticle approach with a Lorentz spectral function. The model parameters are determined by a fit of the entropy $s$ to lattice QCD data. The effective degrees of freedom are found to be rather heavy and of a sizeable width. With the spectral width being closely related to the interaction rate, we find a large effective cross section, which is comparable to the typical distance squared of the quasiparticles. This suggests that the system should be viewed as a liquid as also indicated by an estimate of the plasma parameter $\Gamma$. Furthermore, within the quasiparticle approach we find a very low viscosity to entropy ratio, $\eta / s \sim 0.2$ for $T>1.05 T_{c}$, supporting the recent conjecture of an almost ideal quark-gluon liquid seen at RHIC.

PACS numbers: $12.38 \mathrm{Mh}, 25.75 .-\mathrm{q}$
\end{abstract}

The formation of a quark-gluon plasma (QGP) and its transition to interacting hadronic matter - as occurred in the early universe - has motivated a large community for more than two decades (cf. [1] and Refs. therein). However, the complexity of the dynamics in ultrarelativistic nucleus-nucleus collisions - producing high density matter for short time scales - has not been fully unravelled and many properties of the new phase are still under debate [2]. In central $\mathrm{Au}+\mathrm{Au}$ collisions energy densities are reached at the Relativistic Heavy Ion collider (RHIC) that are far above the critical energy density $e_{c} \sim 1 \mathrm{GeV} / \mathrm{fm}^{3}$ for a phase transition to a QGP as expected from lattice QCD calculations [3]. A strong radial expansion and elliptic flow of hadrons, furthermore, point towards an early generation of pressure and a high interaction rate in the 'new phase' [4].

The latter observables are severely underestimated in conventional string/hadron transport models [5-7], however hydrodynamical approaches do quite well in describing (at midrapidity) the collective properties of the system for low and moderate transverse momenta [8]. The picture thus emerges that the medium created in ultrarelativistic nucleus-nucleus collisions for a couple of $\mathrm{fm} / \mathrm{c}$ interacts more strongly than hadron/string matter, and it exhibits collective properties that resemble those of a liquid of low shear viscosity $\eta[9]$. In fact, viscous hydrodynamical calculations indicate a very low viscosity to entropy ratio, $\eta / s \approx 0.1 \ldots 0.2$ [10]. This picture is substantially different from the expectation of a weakly coupled colored plasma. There is a variety of models that address the properties of this 'new matter'. It might be some kind of i) 'epoxy' [11], i.e. a system of resonant or bound gluonic states with large scattering length, ii) a system of chirally restored mesons, instanton molecules or equivalently giant collective modes [12], iii) a system of colored bound states of quarks $q$ and gluons $g$, i.e. $g q$, $q q, g g$ etc. [13].

In this letter we will provide quantitative arguments that strongly interacting matter in a certain temperature range above $T_{c}$ is in a liquid phase. Our arguments are based on a generalized quasiparticle description of the system taking into account the spectral width $\gamma[14]$ in addition to the quasiparticle mass [15-19]. The model parameters are adjusted to non-perturbative results of lattice calculations. Since the width is closely related to the interaction rate we can then estimate relevant transport properties, such as the effective cross section and the shear viscosity, for temperatures near $T_{c}$. Since lattice 'data' are more precise for quenched QCD we focus here on pure gluonic systems. We expect, however, similar results for full QCD as argued below. The quantities we address are dominated by 'hard' momenta of the order of the temperature $T$. Accordingly, the quasiparticle properties we are interested in are related to the gluon propagator at hard momentum scales. In this line we take into account $d_{g}=2\left(N_{c}^{2}-1\right)=16$ transverse gluons, and neglect Landau-damping contributions as well as the collective longitudinal modes whose spectral strength is suppressed for larger momenta $[15,16]$.

In order to adjust the quasiparticle properties we first consider thermodynamic bulk properties within the $\Phi$-derivable formalism [20], which yields consistent resummed approximations [21]. To leading-loop order, which is expedient for large coupling as argued in [14], the entropy follows directly from the quasiparticle propagator, cf. Ref. [15],

$$
s^{d q p}=-d_{g} \int \frac{d \omega}{2 \pi} \frac{d^{3} p}{(2 \pi)^{3}} \frac{\partial n}{\partial T}\left(\operatorname{Im} \ln \left(-\Delta^{-1}\right)+\operatorname{Im} \Pi \operatorname{Re} \Delta\right),
$$

where $n(\omega)=(\exp (\omega / T)-1)^{-1}$ denotes the Bose distribution function. We note that in the context of Fermi liquid theory a corresponding approximation is called the dynamical quasiparticle (dqp) entropy [22]. In principle, the resummed propagator $\Delta=\left(\omega^{2}-\boldsymbol{p}^{2}-\Pi\right)^{-1}$ is to be calculated from a 1-loop Schwinger-Dyson equation. To proceed at this point, however, we use a physically motivated Ansatz, assuming a Lorentzian spectral function,

$$
\rho(\omega)=\frac{\gamma}{E}\left(\frac{1}{(\omega-E)^{2}+\gamma^{2}}-\frac{1}{(\omega+E)^{2}+\gamma^{2}}\right) .
$$

With the convention $E^{2}(\boldsymbol{p})=\boldsymbol{p}^{2}+M^{2}-\gamma^{2}$, the param- 
eters $M^{2}$ and $\gamma$ are directly related to the real and imaginary parts of the corresponding (retarded) self-energy, $\Pi=M^{2}-2 i \gamma \omega$. At this point we emphasize that the entropy functional (1) is not restricted to 'strict' quasiparticles, i.e., $\gamma$ need not to be small compared to the typical energy. Following the models [18] we parameterize the quasiparticle mass in the gauge invariant and momentum independent asymptotic form

$$
M^{2}=\frac{N_{c}}{6} g^{2} T^{2},
$$

with $N_{c}=3$ and the running coupling

$$
g^{2}(T)=\frac{48 \pi^{2}}{11 N_{c} \ln \left(\lambda\left(T-T_{s}\right) / T_{c}\right)^{2}},
$$

which permits an enhancement near $T_{c}[18,23]$. Likewise, we parameterize the width in the form $\gamma \sim g^{2} T \ln g^{-1}$ [24] or, equivalently, in terms of $M$ [14],

$$
\gamma=\frac{3}{4 \pi} \frac{M^{2}}{T^{2}} T \ln \frac{c}{(M / T)^{2}},
$$

where $c$ is related to the magnetic sector of QCD. We note that $M$ and $\gamma$, as parameterizations of the complexvalued self-energy $\Pi$ at the relevant large momenta near the 'mass shell', are not related by a dispersion relation.

In the upper part of Fig. 1 we compare the lattice results [25] to the quasiparticle entropy; the fitted parameters are $\lambda=2.42, T_{s}=0.46$ and $c=14.4$. We also display the interaction measure $e-3 p$ (the pressure $p$ and the energy density $e$ are evaluated by thermodynamic relations, cf. [18]), which is particularly sensitive to interaction effects. We emphasize that the remarkable agreement with the lattice data is non-trivial because the functional relation between $\gamma$ and $M$ is fixed, cf. (5).

The adjusted quasiparticle mass and width are displayed in the lower part of Fig. 1. The quasiparticles are rather heavy in line with direct lattice calculations [26]. For $T \gtrsim 1.05 T_{c}$ the width is sizeable, reaching more than $50 \%$ of the mass in a large temperature range. The picture of the strongly interacting plasma is, thus, a system of massive excitations with a large collisional width or short mean-free path - opposite to the original concept of narrow quasiparticles. Near $T_{c}$, however, the width is close to zero. Although we have parameterized $\gamma$ in the 'perturbative' form (5) we expect that the inferred temperature dependence is generic: near $T_{c}$ the width has to be small due to the small entropy at $T \approx T_{c}$ [14]. This is in line with a critical slowing down near a phase transition. Away from $T_{c}$ one has to expect a large width due to the strong coupling and increasing reaction rates. The physical processes contributing to the width are then $g g \leftrightarrow g g$ scatterings as well as splitting and fusion reactions $g g \leftrightarrow g$ or $g g \leftrightarrow g g g$ etc. Summing up the elastic and inelastic channel (and neglecting Bose-enhancement for the final states) we end up with the total binary reaction rate

$$
\frac{d N_{\text {coll }}}{d V d t}=\tilde{\operatorname{Tr}}_{P_{1}} \tilde{\operatorname{Tr}}_{P_{2}} 2 \sqrt{\lambda\left(s, P_{1}^{2}, P_{2}^{2}\right)} \sigma_{\text {tot }}\left(P_{1}, P_{2}\right)
$$

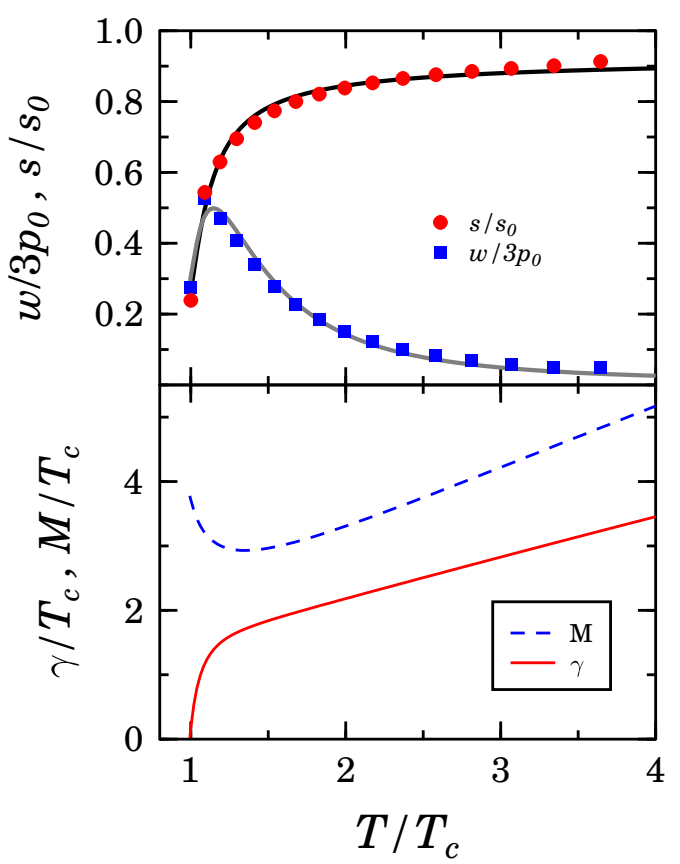

FIG. 1: The entropy $s$ and the interaction measure $w=e-3 p$, in units of the Stefan-Boltzmann limits $s_{0}$ and $p_{0}$, from our quasiparticle model in comparison to lattice calculations [25]. The lower part shows the adjusted mass $M$ and width $\gamma$.

$$
=\langle\sigma\rangle \tilde{\operatorname{Tr}}_{P_{1}} \tilde{\operatorname{Tr}}_{P_{2}} 2 \sqrt{\lambda\left(s, P_{1}^{2}, P_{2}^{2}\right)}=:\langle\sigma\rangle I_{2},
$$

where $\lambda(x, y, z)=(x-y-z)^{2}-4 y z$ and $s=\left(P_{1}+P_{2}\right)^{2}$. In (6) we have introduced the shorthand notation

$$
\tilde{\operatorname{T}} r_{P} \cdots=d_{g} \int \frac{d \omega}{2 \pi} \frac{d^{3} p}{(2 \pi)^{3}} 2 \omega \rho(\omega) \Theta(\omega) \Theta\left(P^{2}\right) n(\omega) \cdots
$$

for the thermally weighted trace over the quasiparticle degrees of freedom. The $\Theta\left(P^{2}\right)$ function ensures that only time-like reaction processes are taken into account in Eq. (6). The interaction rate, on the other hand, is also related to the imaginary part of the self-energy; with a similar factorization as in Eq. (6), $d N_{\text {coll }} / d V d t=\gamma N_{+}$. Here the particle density $N_{+}=\tilde{\operatorname{Tr}} 1$ is the time-like part of the integrated distribution function. The resulting effective total cross section,

$$
\langle\sigma\rangle=\gamma N_{+} / I_{2},
$$

is displayed in the top part of Fig. 2 as a function of $T$ (us$\operatorname{ing} T_{c}=0.26 \mathrm{GeV}$ for quenched QCD). It rises from $\approx 0$ at $T=T_{c}$ to about $20 \mathrm{mb}$ at $T \approx 1.1 T_{c}$, and drops again at higher temperatures. We note that similarly large values for parton cross sections have been used in the phenomenological studies in Ref. [27]. These cross sections are larger by an order of magnitude than typical perturbative estimates for $g g$ scattering (cf. the hatched band in the top part of Fig. 2) where the Debye mass $m_{\text {Debye }}$ is used as an infrared cutoff. However, for strongly coupled 
plasmas the Debye mass may not be the proper regulator as pointed out by Thoma [28].

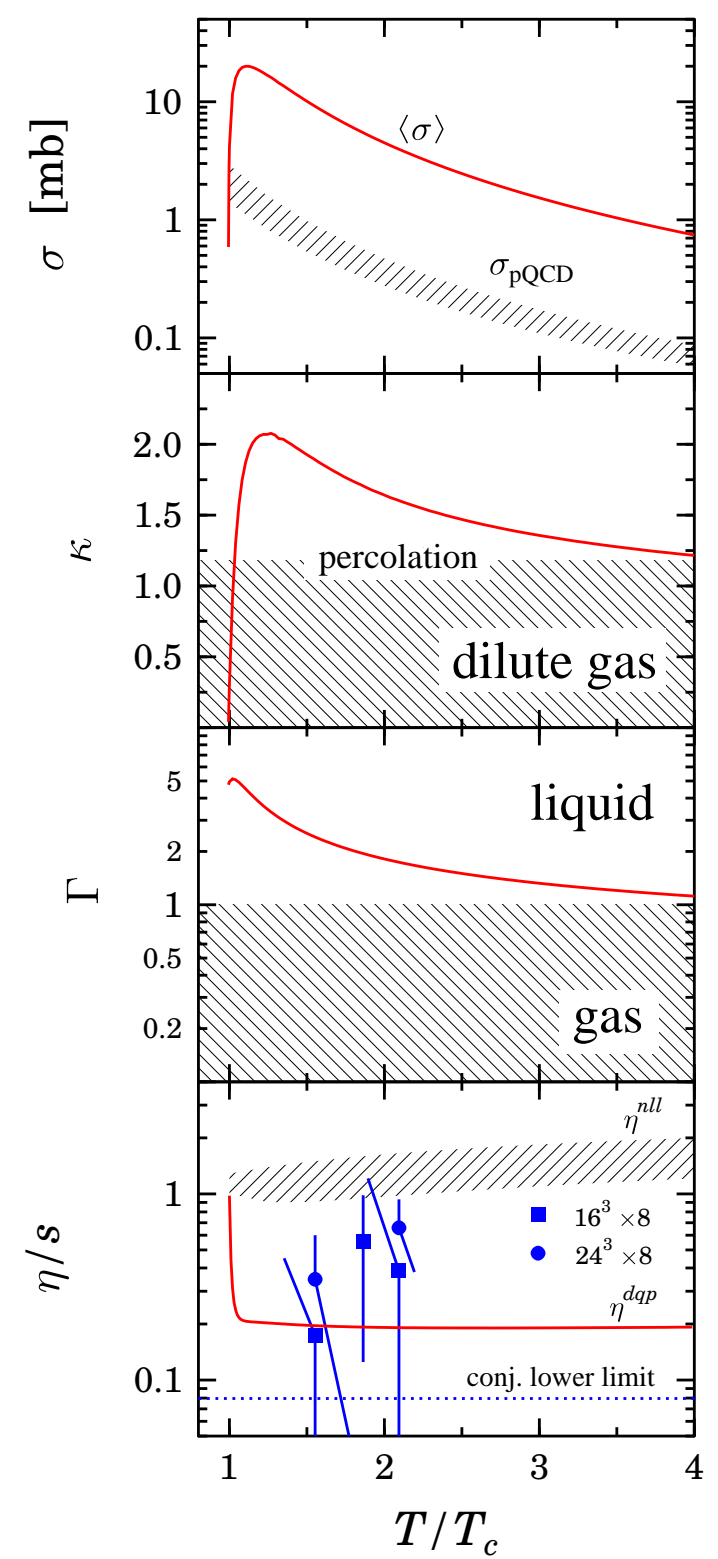

FIG. 2: Upper part: The effective total cross section $\langle\sigma\rangle$, Eq. (8), in comparison to the perturbative estimate $\sigma_{\mathrm{pQCD}}=$ $9 \pi \alpha^{2} / 2 m_{\text {Debye }}^{2}$. 2nd part from top: The percolation measure $\kappa$, Eq. (9); the critical value is $\kappa_{c}=1.18$ [29]. 3rd part from top: The plasma parameter $\Gamma$, Eq. (10). Bottom part: The ratio of shear viscosity to entropy in our quasiparticle model in comparison to the lattice calculation [36] as well as to the next-to-leading $\log$ (nll) result [31]. Note that $\eta / s \approx 0.1 \ldots 0.2$ was estimated from hydrodynamical fits to RHIC data [10].

A question of particular interest is the phase structure of the strongly interacting system, which in Refs. [9, 28] was surmised a liquid. In our quasiparticle approach we can address this question quantitatively by comparing the effective cross section to the typical distance squared of the quasiparticles to obtain information about critical clustering (percolation) [29]. If

$$
\kappa=\langle\sigma\rangle N_{+}^{2 / 3}
$$

is lower than the critical percolation parameter $\kappa_{c} \approx 1.18$ [29] the system is in the kinetic (dilute gas) regime while for $\kappa>\kappa_{c}$ percolation sets in and multi-particle interactions take over as characteristic for a liquid or solid with attractive interactions. As shown in the second part of Fig. $2, \kappa$ is larger than $\kappa_{c}$ for the temperature range $1.05 \ldots 4 T_{c}$. This suggests that the QCD plasma, up to rather large energy densities, is in a liquid/solid phase.

In order to distinguish a liquid from, possibly, a solid phase we consider the plasma parameter [28]. Assuming equal (color) magnetic and electric energies we estimate

$$
\Gamma=2 \frac{N_{c} g^{2}}{4 \pi N^{-1 / 3}} \frac{1}{\left\langle T_{k i n}\right\rangle},
$$

with $N$ being the full particle density (including the space-like contributions), and the average kinetic energy

$$
\left\langle T_{k i n}\right\rangle=N_{+}^{-1} \tilde{\operatorname{Tr}}\left(\omega-\sqrt{p^{2}}\right) .
$$

Empirically it is well established for various attractive interactions that systems with $1 \lesssim \Gamma \lesssim 100$ are in a liquid phase [30]. Since the 'critical' values may slightly vary for different systems we emphasize for the present case that the criteria $\Gamma_{c}=1$ for a gas-liquid phase transition and $\kappa=\kappa_{c}$ for percolation are met at the same temperature, cf. Fig. 2. Thus our results suggest that the gluon plasma is a liquid for temperatures between $T_{c}$ and $4 T_{c}$.

An important property of this gluon liquid is its shear viscosity $\eta$. For weak coupling, it has been calculated in a transport approach from a Boltzmann equation to nextto-leading $\log$ order [31], $\eta^{n l l} \sim T^{3} /\left(g^{4} \ln g^{-1}\right)$. However, for strongly coupled systems such an approach (assuming narrow quasiparticles) might be questionable and the Kubo formalism more appropriate instead [32]. Here the viscosity is evaluated from the slope of the Fourier transform of the spectral function $\left\langle\left[T_{i j}(x), T_{i j}(y)\right]\right\rangle$ for $\omega \rightarrow 0$, where $T_{i j}$ denotes the traceless part of the spatial stress tensor. At 1-loop order (and neglecting the longitudinal contributions) this corresponds directly to our quasiparticle picture which yields, cf. [33],

$$
\eta^{d q p}=-\frac{d_{g}}{60} \int \frac{d \omega}{2 \pi} \frac{d^{3} p}{(2 \pi)^{3}} \frac{\partial n}{\partial \omega} \rho^{2}(\omega)\left[7 \omega^{4}-10 \omega^{2} \boldsymbol{p}^{2}+7 \boldsymbol{p}^{4}\right] .
$$

Note that in the perturbative limit Eq. (12) $\left(\sim T^{4} / \gamma \sim\right.$ $\left.T^{3} /\left(g^{2} \ln g^{-1}\right)\right)$ does not approach $\eta^{\text {nll }} \sim T^{3} /\left(g^{4} \ln g^{-1}\right)$. This comes about as follows: The shear viscosity $\eta$ is inversely proportional to the transport cross section $\sigma_{\text {trans }}$ in which the total scattering rate is weighted by $(1-\cos \theta)$ with $\theta$ denoting the scattering angle [34]. In the weak coupling limit the gluon scattering is strongly forward peaked with a low gain in transverse momentum. This implies that many scatterings, corresponding to ladder 
diagrams in the Kubo formalism, have to be resummed in order to achieve a significant transverse momentum deflection and transport cross section. However, in the case of strong coupling the transport mean-free-path $\lambda_{\text {trans }} \sim 1 /\left(\sigma_{\text {trans }} N_{+}\right)$is expected to become comparable to the total mean-free-path $\lambda \sim 1 /\left(\sigma N_{+}\right)$, as taken into account by Eq. (12). Thus, while a resummation of ladder diagrams is beyond the scope of the quasiparticle model, it should yield a useful approximation near $T_{c}$ [35].

In the bottom part of Fig. 2 we display the ratio of the shear viscosity to entropy. Although the lattice results [36] still have large uncertainties, they are distinctly smaller than the (extrapolation of the) next-to-leading $\log$ result [31]. For $T \gtrsim 1.05 T_{c}$ our quasiparticle result is almost constant, $\eta^{d q p} / s \approx 0.2$ - in good agreement with the estimate $\eta / s \approx 0.1 \ldots 0.2$ from hydrodynamical fits to RHIC data [10]. The fast increase near $T_{c}$, which is related to the characteristic temperature dependence of $\gamma$ and thus also of $\langle\sigma\rangle$, can be seen as a precursor of the phase transition. We mention that the conjectured lower limit [37], $\eta / s \geq 1 /(4 \pi)$, is approximately obtained for $T \approx 1.1 T_{c}$ when calculating $\eta$ in a non-relativistic limit of massive quasiparticles and assuming isotropic transport cross sections [35]. Since $\sigma_{\text {trans }} \leq \sigma$ this estimate should give a lower bound for the viscosity. In conclusion, the quasiparticle model gives the picture of an almost ideal gluon liquid in the relevant temperature range.

The extension of the quasiparticle approach to the physical case (full QCD) is straightforward; besides a change of $T_{c}$, quarks and gluons have the same quasiparticle properties up to group factors [38]. The consequences for ultra-relativistic heavy-ion collisions at RHIC become immediately clear: The large cross sections imply a rapid thermalization of the initial configuration once the initial hard scatterings have produced a high density of minijets. The latter 'pre-equilibrium' processes happen on a scale of $t_{\text {pre }}=2 R_{A} / \gamma \leq 0.14 \mathrm{fm} / \mathrm{c}$ for top RHIC energies such that the equilibration time is essentially governed by the $g g \leftrightarrow g, g g \leftrightarrow g g, g g \leftrightarrow g g g$ and $g g \leftrightarrow g g g g$ processes as also suggested in [39]. After approximate thermalization - of order 0.5 to $1 \mathrm{fm} / \mathrm{c}$ - the system behaves like an almost ideal massive colored parton liquid and exhibits a large pressure. This early pressure is responsible for the transverse flow of hadrons, and the large cross sections result in an almost complete suppression of far-side jets in central collisions.

Acknowledgments: The authors would like to thank C. Greiner, S. Leupold, R. Pisarski, D. Teaney, M. Thoma, and X.N. Wang for helpful discussions and valuable suggestions. This work was supported by BMBF.
[1] Quark Matter 2002, Nucl. Phys. A715 (2003) 1; Quark Matter 2004, J. Phys. G 30 (2004) S633.

[2] Strange Quark Matter 2003, J. Phys. G 30 (2004) 1.

[3] F. Karsch et al., Nucl. Phys. B605 (2001) 579.

[4] H. Weber et al., Phys. Rev. C 67 (2003) 014904.

[5] E.L. Bratkovskaya et al., Phys. Rev. C 67 (2003) 054905; Phys. Rev. C 69 (2004) 054907.

[6] W. Cassing, K. Gallmeister, C. Greiner, Nucl. Phys. A735 (2004) 277.

[7] K. Gallmeister, W. Cassing, Nucl. Phys. A748 (2005) 241.

[8] P. Kolb, U. Heinz, nucl-th/0305084.

[9] E.V. Shuryak, Prog. Part. Nucl. Phys. 53 (2004) 273.

[10] D.A. Teaney, J. Phys. G 30 (2004) S1247.

[11] G.E. Brown, C.-H. Lee, M. Rho, E.V. Shuryak, Nucl. Phys. A740 (2004) 171.

[12] G.E. Brown, C.-H. Lee, M. Rho, Nucl. Phys. A747 (2005) 530.

[13] E.V. Shuryak, I. Zahed, Phys. Rev. D 70 (2004) 054507.

[14] A. Peshier, Phys. Rev. D 70 (2004) 034016.

[15] J. P. Blaizot, E. Iancu, A. Rebhan, Phys. Rev. D 63 (2001) 065003.

[16] A. Peshier, Phys. Rev. D 63 (2001) 105004.

[17] A. Rebhan, P. Romatschke, Phys. Rev. D 68 (2003) 025022 .

[18] A. Peshier, B. Kämpfer, O. P. Pavlenko, G. Soff, Phys. Rev. D 54 (1996) 2399; P. Levai, U. Heinz, Phys. Rev. C 57 (1998) 1879; A. Peshier, B. Kämpfer, G. Soff, Phys. Rev. C 61 (2000) 045203, Phys. Rev. D 66 (2002) 094003.

[19] Yu.B. Ivanov, V.V. Skolov, V.D. Toneev, Phys. Rev. D
$71(2005) 014005$.

[20] J. M. Luttinger, J. C. Ward, Phys. Rev. 118 (1960) 1417.

[21] G. Baym, Phys. Rev. 127 (1962) 1391.

[22] G. M. Carneiro, C. J. Pethick, Phys. Rev. B 11 (1975) 1106.

[23] J. Letessier, J. Rafelski, Phys. Rev. C 67 (2003) 031902.

[24] R. D. Pisarski, Phys. Rev. Lett. 63 (1989) 1129; V. V. Lebedev, A. V. Smilga, Ann. Phys. (N.Y.) 202 (1990) 229.

[25] M. Okamoto et al., Phys. Rev. D 60 (1999) 094510.

[26] P. Petreczky et al., Nucl. Phys. (Proc. Suppl.) B106 (2002) 513.

[27] D. Molnar, M. Gyulassy, Nucl. Phys. A698 (2002) 379.

[28] M.H. Thoma, J. Phys. G 31 (2005) L7; 539.

[29] H. Satz, Nucl. Phys. A661 (1999) 104c.

[30] S. Ichimaru, Rev. Mod. Phys. 54 (1982) 1017.

[31] P. Arnold, G.D. Moore, L.G. Yaffe, JHEP 0305 (2003) 051; 0301 (2003) 030.

[32] B.A. Gelman, E.V. Shuryak, I. Zahed, nucl-th/0410067.

[33] G. Aarts, J.M. Martinez Resco, JHEP 0204 (2002) 053; JHEP 0402 (2004) 061.

[34] M.H. Thoma, Phys. Rev. D49 (1994) 451.

[35] A. Peshier, W. Cassing (to be published).

[36] A. Nakamura, S. Sakai, Phys. Rev. Lett. 94 (2005) 072305.

[37] P. Kovtun, D.T. Son, A.O. Starinets, Phys. Rev. Lett. 94 (2005) 111601.

[38] A. Peshier, J. Phys. G 31 (2005) S371.

[39] Z. Xu, C. Greiner, hep-ph/0406278. 